PROCEEDINGS OF THE

AMERICAN MATHEMATICAL SOCIETY

Volume 138, Number 10, October 2010, Pages 3651-3655

S 0002-9939(10)10462-6

Article electronically published on May 10, 2010

\title{
THE CORONA PROBLEM WITH TWO PIECES OF DATA
}

\author{
STEVEN G. KRANTZ
}

(Communicated by Mei-Chi Shaw)

\begin{abstract}
We study the corona problem on the unit ball in $\mathbb{C}^{n}$, and more generally on strongly pseudoconvex domains in $\mathbb{C}^{n}$. When the corona problem has just two pieces of data, and an extra geometric hypothesis is satisfied, then we are able to solve it.
\end{abstract}

\section{INTRODUCTION}

Banach algebras were created in the thesis of I. M. Gelfand in 1938. Some of the key ideas in the subject are recorded in [9] and [10. In 1942, S. Kakutani formulated the following fundamental question (known as the corona problem) in the subject:

Suppose that $f_{1}, \ldots, f_{k}$ are bounded, holomorphic functions on the unit disc $D$ with the property that

$$
\left|f_{1}(\zeta)\right|+\left|f_{2}(\zeta)\right|+\cdots+\left|f_{k}(\zeta)\right|>\delta>0
$$

for some positive constant $\delta$ and all $\zeta \in D$. [We call $f_{1}, \ldots, f_{k}$ a set of corona data on the disc.] Do there exist bounded, holomorphic $g_{j}, j=1, \ldots, k$, on $D$ such that

$$
f_{1}(\zeta) \cdot g_{1}(\zeta)+f_{2}(\zeta) \cdot g_{2}(\zeta)+\cdots+f_{k}(\zeta) \cdot g_{k}(\zeta) \equiv 1
$$

for all $\zeta \in D$ ?

It turns out that this question is equivalent to asking whether the point evaluation functionals are weak-* dense in the space of multiplicative linear functionals on the Banach algebra $H^{\infty}(D)$. This equivalence is explained in some detail in [14]. An authoritative treatment of the corona problem on the disc appears in [7].

Lennart Carleson 3 solved the corona problem on the disc $D$ in the affirmative in 1962. Since that time, the corona problem has been proved to have a positive solution on a variety of different types of domains in $\mathbb{C}$. See, for example, [8]. Brian Cole produced a Riemann surface on which the corona problem fails (see [6] and [7] for a discussion).

Received by the editors January 12, 2010.

2010 Mathematics Subject Classification. Primary 30H80, 32A38, 32A65.

Key words and phrases. Corona problem, bounded holomorphic functions, domain of holomorphy, ball.

The author was supported in part by the National Science Foundation and by the Dean of the Graduate School at Washington University.

(C)2010 American Mathematical Society
ublic domain 28 years from publication Reverts to public domain 28 years from publication 
Meanwhile, there have been investigations of the corona problem on domains in $\mathbb{C}^{n}$, particularly on the ball and the polydisc. A variety of counterexamples to the corona problem in several variables have been produced in [18] and [5].

At this time there is no known domain in the plane $\mathbb{C}$ on which the corona problem is known to fail, and there is no known domain in $\mathbb{C}^{n}$, for $n \geq 2$, on which the corona problem is known to hold true. Some partial results on the corona problem in several complex variables appear, for instance, in [15], [16, [17, and [2].

Results that are philosophically related to those in this paper appear in [4. It is a pleasure to thank Urban Cegrell for useful discussions.

\section{Fundamental Results}

In the present paper we study the corona problem on the ball in $\mathbb{C}^{n}$, and more generally on strongly pseudoconvex domains in $\mathbb{C}^{n}$, when there are just two pieces of corona data (i.e., $k=2$ ). Using the Koszul complex (an idea developed for the computation of the cohomology of a Lie algebra), we are able to produce a solution of the corona problem in these circumstances. Afterwards we shall make some remarks about the case when there are an arbitrary (but, of course, finite) number of pieces of corona data.

We shall work on a domain $\Omega$ in $\mathbb{C}^{n}$, where here a domain is a connected, open set.

We take this opportunity to review how the Koszul complex works in the present context. Refer, for instance, to [13] or [14] for more information about the Koszul complex.

If $f_{1}, f_{2}, \ldots, f_{k}$ are given corona data on a domain $\Omega \subseteq \mathbb{C}^{n}$, we define

$$
U_{j}=\left\{\zeta \in \Omega:\left|f_{j}(\zeta)\right|>\frac{\delta}{2 k}\right\} .
$$

It is easy to see then that the $U_{j}$ are open and $\bigcup_{j} U_{j} \supseteq \Omega$. Let $\left\{\varphi_{j}\right\}$ be a partition of unity subordinate to the covering $\left\{U_{j}\right\}$.

Now set

$$
g_{j}=\frac{\varphi_{j}}{f_{j}}+\sum v_{j i} f_{i},
$$

where the $v_{j i}$ are functions yet to be determined and $v_{i j}=-v_{j i}$ and $v_{j j} \equiv 0$. Then it is a simple formal verification to see that the $g_{j}$ are well-defined and

$$
\sum_{j} f_{j} g_{j} \equiv 1 \text {. }
$$

Now our main result is this:

Theorem 1. Let $\Omega \subseteq \mathbb{C}^{n}$ be strongly pseudoconvex. Let $f_{1}, f_{2}$ be corona data on $\Omega$. Let $\mathcal{Z}_{1}=\left\{z \in \Omega: f_{1}(z)=0\right\}$ and $\mathcal{Z}_{2}=\left\{z \in \Omega: f_{2}(z)=0\right\}$. Assume that there is an $\eta>0$ such that, if $z \in \mathcal{Z}_{1}$ and $w \in \mathcal{Z}_{2}$, then $|z-w|>\eta$. Then there exist bounded, holomorphic $g_{1}, \ldots, g_{k}$ such that

$$
f_{1} g_{1}+f_{2} g_{2} \equiv 1 \text {. }
$$

Proof. Let us return to the Koszul complex. Because the $\varphi_{j}$ are real functions, we see immediately that the $g_{j}$ so defined are not necessarily holomorphic. But we would like to see that it is possible to choose $v_{j i}$ so that each $g_{j}$ will in fact be holomorphic. We may choose $U_{1}, U_{2}$ so that their boundaries are tangent to 
some high order $m$ where they cross. As a result, we may choose $\varphi_{2}$ so that $\bar{\partial} \varphi_{2}$ is nonzero only on $U_{1} \cap U_{2}$ and $\left|\nabla \varphi_{2}\right| \leq C \cdot \delta(z)^{-1 / m}$, where $\delta(z)$ is the distance of $z$ to $\partial\left(U_{1} \cap U_{2}\right)$. Therefore $\nabla \varphi_{2} \in L^{2 n+3}(\Omega)$ when $m$ is large. This fact (using results of [12]) will be crucial below.

The holomorphicity entails

$$
\bar{\partial} g_{j}=\frac{\bar{\partial} \varphi_{j}}{f_{j}}+\sum\left(\bar{\partial} v_{j i}\right) f_{i} \equiv 0 .
$$

In general the algebra involved in understanding this last equation is rather complicated. But we take $k=2$. Then we find the situation to be tenable. Namely, we may write

$$
\bar{\partial} v_{11} f_{1}+\bar{\partial} v_{12} f_{2}=-\frac{\bar{\partial} \varphi_{1}}{f_{1}}
$$

and

This simplifies to

$$
\bar{\partial} v_{21} f_{1}+\bar{\partial} v_{22} f_{2}=-\frac{\bar{\partial} \varphi_{2}}{f_{2}}
$$

$$
\bar{\partial} v_{12} f_{2}=-\frac{\bar{\partial} \varphi_{1}}{f_{1}}
$$

and

$$
\bar{\partial} v_{21} f_{1}=-\frac{\bar{\partial} \varphi_{2}}{f_{2}} .
$$

Using the given identities on the $v_{j i}$, we then find that

$$
\bar{\partial} v_{12} f_{2}=-\frac{\bar{\partial} \varphi_{1}}{f_{1}}
$$

and

$$
-\bar{\partial} v_{12} f_{1}=-\frac{\bar{\partial} \varphi_{2}}{f_{2}}
$$

Since $\varphi_{1}+\varphi_{2} \equiv 1$, we know that $\bar{\partial} \varphi_{1}=-\bar{\partial} \varphi_{2}$. So in fact the last two equations say the same thing:

$$
\bar{\partial} v_{12}=\frac{\bar{\partial} \varphi_{2}}{f_{1} f_{2}} \equiv \lambda .
$$

This last is the $\bar{\partial}$-problem that we must solve. First notice that the right-hand side is $\bar{\partial}$-closed. So the problem can be solved on a pseudoconvex domain (see [13]).

Now we have assumed that the zero set of $f_{1}$ is bounded away from the zero set of $f_{2}$. That is, there is an $\eta>0$ so that, if $f_{1}(z)=0$ and $f_{2}(w)=0$, then $|z-w|>\eta$. (Unfortunately, the corona condition does not guarantee this assumption. It does guarantee that the assumption holds on the smaller disc $\bar{D}(0, r), 0<r<1$, but of course the estimate on $\lambda$ does not hold uniformly in $r$.)

As previously noted, we have that $\bar{\partial} \varphi_{2}$ is nonzero only when $\left|f_{1}\right| \geq \delta /(2 k)$ and $\left|f_{2}\right| \geq \delta /(2 k)$. Also $\bar{\partial} \varphi_{2} \in L^{2 n+3}(\Omega)$.

As a consequence, the data function on the right-hand side of $(*)$ is in $L^{2 n+3}(\Omega)$, and we may solve (using results of [12]) to obtain a bounded $v_{12}$. Therefore $g_{1}$ and $g_{2}$, according to $(\star)$, are bounded functions, and we have solved the corona problem for the data $f_{1}, f_{2}$. 
Remark 2. An essential part of the argument just presented is that the zero sets of $f_{1}$ and $f_{2}$ are separated. One may wonder to what degree this sort of property can be guaranteed. In particular, is it possible for a bounded holomorphic $h$ on the ball $B$ to have zero set accumulating at every boundary point (if so, two such functions could not have their zero sets separated).

In dimension 1 , the answer to this last question is easily "yes". Let $\Omega=D$ be the unit disc, and let $\mathcal{S}$ be the set consisting of

- two evenly spaced points at distance $2^{-2}$ from the boundary of $D$;

- three evenly spaced points at distance $2^{-3}$ from the boundary of $D$;

- four evenly spaced points at distance $2^{-4}$ from the boundary of $D$;

- $\quad$...

- $k$ evenly spaced points at distance $2^{-k}$ from the boundary of $D$.

Then the Blaschke product with zero set $\mathcal{S}$ will have the desired property.

In higher dimensions it is more difficult to construct such a function, but it can be done. The paper 1 gives the tools for producing an example on the ball $B$ in complex dimension 2. Indeed, Berndtsson shows that any analytic set of bounded volume is the zero set of a bounded function. Thus one may take $\left\{p_{j}\right\}$ to be a countable, dense set in $\partial B$, and then let $\left\{\mathbf{d}_{j k}\right\}$ be analytic discs of radius $2^{-j+k}$, $k=1,2, \ldots$, which lie in the domain and approach $p_{j}$. Then the union of all these discs is an analytic set with finite 2 -dimensional volume and is thus the zero set of an $H^{\infty}$ function; that function has the properties that we seek. Also note that the paper 11] gives an example on $B$ in all dimensions; indeed, Hakim and Sibony produce a bounded, holomorphic function whose zeros accumulate (but not admissibly!) at each boundary point, but which is not the identically zero function.

\section{Concluding Remarks}

The corona problem is an important part of classical function theory, and of modern harmonic analysis. The present paper offers the first positive result in several complex variables that is in the spirit of the original corona problem. We hope in future papers to extend the result to an arbitrary number of pieces of corona data.

\section{REFERENCES}

[1] B. Berndtsson, Integral formulas for the $\bar{\partial}$-equation and zeros of bounded holomorphic functions in the unit ball, Math. Ann. 249(1980), 163-176. MR578723 (81m:32012)

[2] B. Berndtsson, S. Y. Chang, and K. C. Lin, Interpolating sequences in the polydisc, Trans. Amer. Math. Soc. 302(1987), 161-169. MR887503 (88i:32011)

[3] L. Carleson, Interpolation by bounded analytic functions and the corona problem, Ann. of Math. (2) 76(1962), 547-559. MR0141789(25:5186)

[4] U. Cegrell and A. Fällström, Spectrum of certain Banach algebras and $\bar{\partial}$-problems, Ann. Polon. Math. 90(2007), 51-58. MR2283112 (2008d:32009)

[5] J. E. Fornæss and N. Sibony, Smooth pseudoconvex domains in $\mathbb{C}^{2}$ for which the corona theorem and $L^{p}$ estimates for $\bar{\partial}$ fail, Complex Analysis and Geometry, 209-222, Univ. Ser. Math., Plenum, New York, 1993. MR1211882 (94a:32028)

[6] T. W. Gamelin, Uniform Algebras, Prentice-Hall, Englewood Cliffs, NJ, 1969. MR0410387 $(53: 14137)$

[7] J. B. Garnett, Bounded Analytic Functions, Academic Press, New York, 1981. MR628971 (83g:30037)

[8] J. B. Garnett and P. W. Jones, The corona theorem for Denjoy domains, Acta Math. 155(1985), 27-40. MR793236 (87e:30044) 
[9] I. M. Gelfand, To the theory of normed rings. II. On absolutely convergent trigonometrical series and integrals, C. R. (Doklady) Acad. Sci. URSS (N.S.) 25(1939), 570-572. MR0001984 $(1: 330 f)$

[10] I. M. Gelfand, To the theory of normed rings. III. On the ring of almost periodic functions, C. R. (Doklady) Acad. Sci. URSS (N.S.) 25(1939), 573-574. MR0001985 (1:331a)

[11] M. Hakim and N. Sibony, Fonctions holomorphes bornées et limites tangentielles, Duke Math. Journal 50(1983), 133-141. MR700133 (84m:32011)

[12] S. G. Krantz, Optimal Lipschitz and $L^{p}$ regularity for the equation $\bar{\partial} u=f$ on strongly pseudo-convex domains, Math. Annalen 219(1976), 233-260. MR0397020(53:880)

[13] S. G. Krantz, Function Theory of Several Complex Variables, $2^{\text {nd }}$ ed., American Mathematical Society, Providence, RI, 2001. MR 1846625 (2002e:32001)

[14] S. G. Krantz, Cornerstones of Geometric Function Theory: Explorations in Complex Analysis, Birkhäuser Publishing, Boston, 2006. MR2167675 (2006e:30001)

[15] S. G. Krantz and S. Y. Li, Some remarks on the corona problem on strongly pseudoconvex domains in $\mathbb{C}^{n}$, Illinois J. Math. 39(1995), 323-349. MR1316541 (96g:32014)

[16] S. G. Krantz and S. Y. Li, Explicit solutions for the corona problem with Lipschitz data in the polydisc, Pacific J. Math. 174(1996), 443-458. MR.1405596 (97h:46088)

[17] S. G. Krantz and S. Y. Li, Factorization of functions in subspaces of $L^{1}$ and applications to the corona problem, Indiana Univ. Math. J. 45(1996), 83-102. MR.1406685 (97h:46089)

[18] N. Sibony, Problème de la couronne pour des domaines pseudoconvexes à bord lisse, Ann. of Math. (2) 126(1987), 675-682. MR916722 (88k:32012)

Department of Mathematics, Washington University in St. Louis, St. Louis, Missouri 63130

E-mail address: sk@math.wustl.edu 